INPLASY

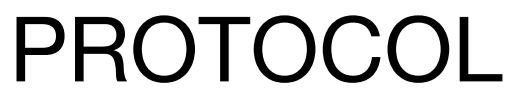

To cite: Tian et al. Association Between Prior Antiplatelet Therapy and Prognosis in Patients with Intracerebral Hemorrhage. Inplasy protocol 202080015. doi:

10.37766/inplasy2020.8.0015

Received: 05 August 2020

Published: 05 August 2020

Corresponding author:

Ye Tian

tianye030710@163.com

Author Affiliation:

Tianjin Medical University

General Hospital

Support: None.

Review Stage at time of this submission: Formal screening of search results against eligibility criteria.

Conflicts of interest:

None.

\section{Association Between Prior Antiplatelet Therapy and Prognosis in Patients with Intracerebral Hemorrhage}

Tian, Y1; Zhang, J2; Yuan, $\mathrm{H}^{3}$; Jiang, R4; Zhang, J5.

Review question / Objective: Antiplatelet therapy (APT) promotes bleeding, but brain has more pro-coagulant factors. Therefore, APT might not worsen outcome in patients with intracerebral hemorrhage (ICH). This study will investigate the association between prior APT use and prognosis of patients with ICH.

Condition being studied: ICH is the most disastrous stroke subtype. Prognosis is considered worse with prior antithrombotic treatment. This study will investigate the association between prior APT use and prognosis of patients with ICH.

INPLASY registration number: This protocol was registered with the International Platform of Registered Systematic Review and Meta-Analysis Protocols (INPLASY) on 05 August 2020 and was last updated on 05 August 2020 (registration number INPLASY202080015).

\section{INTRODUCTION}

Review question / Objective: Antiplatelet therapy (APT) promotes bleeding, but brain has more pro-coagulant factors. Therefore, APT might not worsen outcome in patients with intracerebral hemorrhage (ICH). This study will investigate the association between prior APT use and prognosis of patients with ICH.

Condition being studied: $\mathrm{ICH}$ is the most disastrous stroke subtype. Prognosis is considered worse with prior antithrombotic treatment. This study will investigate the 
association between prior APT use and prognosis of patients with ICH.

METHODS

Search strategy: We did electronic search in MEDLINE, EMBASE, and Web of Science.

Participant or population: Patients without age limit who were diagnosed with $\mathrm{ICH}$ and had a medical history of APT use prior to onset of ICH were included. The duration of prior therapy was not limited. Patients with subarachnoid hemorrhage or secondary ICH due to trauma, tumor, ruptured aneurysm, or arteriovenous malformations were excluded.

Intervention: APTs included aspirin, clopidogrel, ticlopidine, ticagrelor, cangrelor, sarpogrelate, etc. The APTs could be used single or in combination. There were no limitation on dosage, treatment duration, frequency and administration methods. APTs should be used prior to the occurrence of ICH.

Comparator: No APT, or single/combined aspirin/clopidogrel.

Study designs to be included: Any observational study design were included.

Eligibility criteria: We included studies comparing APT with no APT, or studies comparing single/combined aspirin/ clopidogrel.

Information sources: We did electronic search in MEDLINE, EMBASE, and Web of Science. These databases were searched from inception to January 2020. In case some data we need were not reported in the publications of included studies, we will try to contact with the authors.

Main outcome(s): - All-cause mortality (ICU/in-hospital mortality, measured at discharge, 30 days and later, etc.); • Hemorrhage expansion, with more than $33 \%$ or $12.5 \mathrm{~mL}$ increase at follow-up hematoma volume compared to the baseline, within 4 days; - Modified Rankin Scale (mRS) score, to measure neurological disability, classification defined by original studies, at hospital discharge, 30 days and later.

Quality assessment / Risk of bias analysis: We will use the QUIPS tool to assess the risk of bias of included studies. We will assess each study's risk of bias considering six domains: participation, attrition, prognostic factor measurement, confounding measurement and account, outcome measurement, and statistical analysis and reporting.

Strategy of data synthesis: For risk factor studies, meta-analysis will only be performed should studies be judged homogenous in terms of characteristics of population, prior APT use, outcomes and study design, and statistical metric. Otherwise, data will be synthesized narratively. We will use random-effect method for all analyses. We will conduct separate meta-analyses of RRs, ORs, HRs (at similar follow-up points) and RDs, and unadjusted and adequately adjusted results.

Subgroup analysis: Where data is available, we will conduct subgroup analysis to test the difference in effect size for the following subgroups: - Different APT drugs used (for those studies included subgroup outcomes on different APT drugs); • Different mRS score classifications (e.g. subgroup analysis of $\mathrm{mRS} 0-3 / 4-6$ ).

Sensibility analysis: None.

Language: English.

Country(ies) involved: China.

Keywords: APT; ICH; prognosis; aspirin; clopidogrel.

Contributions of each author:

Author 1 - Ye Tian.

Author 2 - Jiacheng Zhang.

Author 3 - Hengjie Yuan.

Author 4 - Rongcai Jiang.

Author 5 - Jianning Zhang. 